\title{
Is it time to choose amiodarone for postoperative atrial fibrillation?
}

Adam E. Saltman, MD

See related article on page 1420.

From the Department of Surgery, Division of Cardiothoracic Surgery, University of Massachusetts Memorial Medical Center, Worcester, Mass.

Received for publication Dec 3, 2002; accepted for publication Dec 16, 2002.

Address for reprints: A. E. Saltman, MD, Department of Surgery, Division of Cardiothoracic Surgery, University of Massachusetts Memorial Medical Center, 55 Lake Ave N S3-747, Worcester, MA 01655 (Email: aesmdphd@hotmail.com).

J Thorac Cardiovasc Surg 2003;125:1202-3

Copyright () 2003 by The American Association for Thoracic Surgery

0022-5223/2003\$30.00+0

doi:10.1016/S0022-5223(03)00224-1

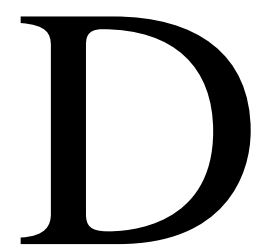

espite the astonishing advances that have been made in cardiac surgery over the past 40 years, new-onset atrial fibrillation remains its most common complication. Long thought a nuisance, it has now been clearly shown to increase length of stay, intensive care unit utilization, morbidity, and even long-term mortality. It occurs in anywhere from $15 \%$ to $40 \%$ of patients and little progress has been made in our understanding, prevention, or treatment of it.

Yagdi and colleagues ${ }^{1}$ have presented elsewhere in this issue of the Journal their study on the use of amiodarone to prevent new-onset postoperative atrial fibrillation (PAF) in patients undergoing coronary artery bypass grafting. They studied a group of 157 subjects, randomly assigned to receive amiodarone after surgery or their usual care without amiodarone. The drug was administered as an intravenous infusion over 2 days, beginning immediately after surgery, followed by declining oral dosing over a 30-day period. The investigators concluded that the postoperative administration of amiodarone was effective at significantly reducing the incidence of PAF by $14.6 \%$, the average duration of PAF episodes by 21.9 hours, the average ventricular response rate by 20 beats/min, and the average hospital length of stay by 1 day.

This excellent study provides additional substance to the growing body of literature showing that amiodarone is an effective agent at reducing the incidence of $\mathrm{PAF}^{2-5}$ although not every study so far published agrees with this conclusion. ${ }^{6}$ What is not yet known, however, is whether amiodarone is a superior drug at preventing PAF when compared with more standard lines of prophylaxis such as $\beta$-blockers.

To show that amiodarone should be the drug of choice against PAF, one must address two issues: First, is amiodarone actually more potent than other drugs at reducing the incidence of PAF, particularly in comparison with the inexpensive $\beta$-blockers? Second, is amiodarone cost-effective at reducing PAF, particularly considering its expensive intravenous form?

There is actually very little information currently available addressing the potency of amiodarone relative to other drugs, and what is available is fairly negative. Wurdeman and colleagues ${ }^{7}$ recently performed a meta-analysis of the literature concerning amiodarone versus sotalol and found that there was no difference in the incidence of PAF or atrial flutter, length of hospital stay, or adverse drug reaction between the drugs. Tokmakoglu and coworkers ${ }^{8}$ tested amiodarone against digoxin and metoprolol in a randomized trial and found that both regimens proved effective at reducing the incidence of PAF. Although PAF was less frequent in the amiodarone arm of this study ( $8 \%$ vs $16 \%$ ), there was no statistical difference between these two groups. In a study of amiodarone against propanolol, Solomon and colleagues ${ }^{9}$ were able to show that amiodarone was superior with regard to preventing PAF but not with regard to length of stay. In the AMIBLOCK trial, the preliminary results of which were recently presented in abstract form, amiodarone was shown to be less effective and less safe than atenolol. ${ }^{10}$

The second question, cost-effectiveness, proves to be even more poorly answered at this point. Referring to the Wurdeman study, ${ }^{7}$ a quick calculation reveals that generic oral sotalol given at $120 \mathrm{mg}$ daily for 7 days would cost about $\$ 25$. 
Intravenous amiodarone costs $\$ 421$ for a 150 -mg ampule and \$1264 for a 450-mg infusion; patient charges would typically be about 5 times higher. An intravenous 150-mg loading dose of amiodarone followed by a 7-day oral course of $1000 \mathrm{mg}$ daily would cost approximately $\$ 475$. Mahoney and colleagues ${ }^{11}$ recently showed that amiodarone did not prove cost-effective at reducing PAF except in older patients, particularly those with chronic lung disease (interestingly, a group in which most practitioners would be reluctant to prescribe amiodarone).

Therefore, although it is clear that amiodarone reduces the incidence of PAF, it has not yet been determined whether it is more potent or cost-effective than $\beta$-blockers or other class III antiarrhythmic drugs already in use. (In fact, because amiodarone does possess some $\beta$-blocking effect, it is possible that this is the mechanism by which it exerts its salutary actions.) As a measure of relief, one study has shown that an inexpensive 7-day preoperative oral loading regimen was effective at reducing $\mathrm{PAF}^{2}$; however, few surgeons have the luxury of meeting their patients 7 days before a cardiac operation!

Therefore, until more convincing evidence emerges, the routine administration of $\beta$-blockers remains standard prophylaxis for PAF. Amiodarone should be reserved for those patients in whom $\beta$-blockade would be contraindicated or who poorly tolerate the drug, such as those with poor ventricular function, congestive heart failure, severe lung disease, thyroid disease, or allergy.

\section{References}

1. Yagdi T, Nalbantgil S, Ayik F, et al. Amiodarone reduces the incidence of atrial fibrillation after coronary artery bypass grafting. $J$ Thorac Cardiovasc Surg 203;125:1420-5.

2. Daoud EG, Strickberger SA, Man KC, et al. Preoperative amiodarone as prophylaxis against atrial fibrillation after heart surgery. $N$ Engl J Med. 1997;337:1785-91.

3. Redle JD, Khurana S, Marzan R, et al. Prophylactic oral amiodarone compared with placebo for prevention of atrial fibrillation after coronary artery bypass surgery. Am Heart $J .1999 ; 138: 144-50$.

4. Giri S, White CM, Dunn AB, et al. Oral amiodarone for prevention of atrial fibrillation after open heart surgery, the atrial fibrillation suppression trial (AFIST): a randomised placebo-controlled trial. Lancet. 2001;357:830-6.

5. Katariya K, DeMarchena E, Bolooki H. Oral amiodarone reduces incidence of postoperative atrial fibrillation. Ann Thorac Surg. 1999; 68:1599-603.

6. Maras D, Boskovic SD, Popovic Z, et al. Single-day loading dose of oral amiodarone for the prevention of new-onset atrial fibrillation after coronary artery bypass surgery. Am Heart J. 2001;141:E8.

7. Wurdeman RL, Mooss AN, Mohiuddin SM, Lenz TL. Amiodarone vs sotalol as prophylaxis against atrial fibrillation after heart surgery: a meta-analysis. Chest. 2002;121:1203-10.

8. Tokmakoglu H, Kandemir O, Gunaydin S, Catav Z, Yorgancioglu C, Zorlutuna Y. Amiodarone versus digoxin and metoprolol combination for the prevention of postcoronary bypass atrial fibrillation. Eur J Cardiothorac Surg. 2002;21:401-5.

9. Solomon AJ, Greenberg MD, Kilborn MJ, Katz NM. Amiodarone versus a $\beta$-blocker to prevent atrial fibrillation after cardiovascular surgery. Am Heart J. 2001;142:811-5.

10. Zillo AC, de Zuloaga C, Guastavino D, et al. AMIBLOCK trial: amiodarone vs. beta-blockers for prevention of post CABG atrial tachyarrhythmias (abstract). Circulation. 2002;106(Suppl):II-605.

11. Mahoney EM, Thompson TD, Veledar E, Williams J, Weintraub WS. Cost-effectiveness of targeting patients undergoing cardiac surgery for therapy with intravenous amiodarone to prevent atrial fibrillation. $J \mathrm{Am}$ Coll Cardiol. 2002;40:737-45. 